\title{
Short-form measures of diabetes-related emotional distress: the Problem Areas in Diabetes Scale (PAID)-5 and PAID-1
}

\author{
B. E. McGuire • T. G. Morrison • N. Hermanns • S. Skovlund • E. Eldrup • \\ J. Gagliardino • A. Kokoszka • D. Matthews • M. Pibernik-Okanović • \\ J. Rodríguez-Saldaña $\cdot$ M. de Wit $\cdot$ F. J. Snoek
}

Received: 9 June 2009 /Accepted: 14 September 2009 / Published online: 20 October 2009

(C) Springer-Verlag 2009

\begin{abstract}
Aims/hypothesis We wanted to identify a five-item short form of the Problem Areas in Diabetes Scale and a single-item measure for rapid screening of diabetes-related emotional distress.

Methods Using an existing database of 1,153 patients with diabetes, we conducted a principal-components analysis to identify a set of five items and then conducted a reliability analysis and validity checks. From those five items, we identified the item with the strongest psychometric properties as a one-item screening tool.

Results We identified a reliable and valid short version of the Problem Areas in Diabetes Scale (PAID)
\end{abstract}

Electronic supplementary material The online version of this article (doi:10.1007/s00125-009-1559-5) contains supplementary material, which is available to authorised users.

B. E. McGuire $(\bowtie)$

Clinical Psychology Programme,

School of Psychology,

National University of Ireland,

Galway, Ireland

e-mail: brian.mcguire@nuigalway.ie

T. G. Morrison

Department of Psychology,

University of Saskatchewan,

Saskatoon, Saskatchewan,

Canada

N. Hermanns

Diabetes Klinik,

Bad Mergentheim, Germany

S. Skovlund

Novo Nordisk,

Copenhagen, Denmark

E. Eldrup

STENO Diabetes Centre,

Copenhagen, Denmark comprising five of the emotional-distress questions of the full PAID items (PAID-5, with items 3, 6, 12, 16, 19). The PAID-5 has satisfactory sensitivity (94\%) and specificity $(89 \%)$ for recognition of diabetes-related emotional distress. We also identified a one-item screening tool, the PAID-1 (Question 12: Worrying about the future and the possibility of serious complications), which has concurrent sensitivity and specificity of about $80 \%$ for the recognition of diabetes-related emotional distress.

Conclusions/interpretation The PAID-5 and PAID-1 appear to be psychometrically robust short-form measures of diabetes-related emotional distress.

\footnotetext{
J. Gagliardino

Centre for Experimental and Applied Endocrinology,

National University La Plata,

La Plata, Argentina

A. Kokoszka

II Department of Psychiatry, Medical University of Warsaw,

Warsaw, Poland

D. Matthews

NIHR Oxford Biomedical Research,

Oxford, UK

M. Pibernik-Okanović

VUK Vrhovac University Clinic,

Zagreb, Croatia

J. Rodríguez-Saldaña

Diabetes Program,

Hidalgo, Mexico

M. de Wit $\cdot$ F. J. Snoek

VU Medical Center,

Amsterdam, The Netherlands
} 
Keywords Diabetes $\cdot$ Distress $\cdot$ Emotional $\cdot$ Measurement . Problem areas in diabetes scale

$\begin{array}{ll}\text { Abbreviations } \\ \text { EFA } & \text { Exploratory factor analysis } \\ \text { PAID } & \text { Problem Areas in Diabetes Scale } \\ \text { PAID-1 } & \begin{array}{l}\text { Problem Areas in Diabetes Scale_-One-item } \\ \text { Screening Form }\end{array} \\ \text { PAID-5 } & \begin{array}{l}\text { Problem Areas in Diabetes Scale_-Five-item } \\ \text { Short Form }\end{array} \\ \text { WHO-5 } & \text { WHO Five Item Measure of Wellbeing }\end{array}$

The prevalence of serious psychological distress in people with diabetes is significant and contributes to diseaserelated burden [1]. Consequently, international guidelines advocate routine screening for psychological problems $[2,3]$. The Problem Areas in Diabetes Scale (PAID) [4] has been recommended for identifying depression and diabetes-related distress [5]. The PAID is a widely used measure, containing 20 items which have a five-point response option ( $0-4$ representing 'Not a problem' through to 'Serious problem'). The PAID has four factors or dimensions measuring diabetes-related emotional problems (12 questions), treatment-related problems (three questions), food-related problems (three questions), and social support-related problems (two questions). The PAID has been translated into various languages, is widely employed to monitor change following an intervention and its psychometric properties have been established [6, 7]. However, while clinically useful, wider use of the PAID may be limited by its length.

Recently, a two-item screening version of the Diabetes Distress Scale (DDS) was validated and showed good sensitivity (95\%) and specificity (85\%) [8]. However, as the PAID remains a more widely used instrument, we aimed to develop a five-item short form for routine clinical and research use and a single-item measure that may be used as a rapid screen for diabetes-related emotional distress.

\section{Methods}

Participants Our sample comprised 1,153 respondents to the PAID from an ongoing international, multicentre study of psychosocial care in diabetes (Diabetes Attitudes Wishes and Needs [DAWN] Monitoring Individual Needs in Diabetes [MIND] study) [9]. Data on well-being (WHO Five Item Measure of Wellbeing [WHO-5]) and on various demographic and biomedical variables are also included.

The average age of respondents was 53.8 years $(S D=$ 14.7; range $=18-89), 601(52.1 \%)$ were women, and most were employed full-time $(36.8 \%, n=424)$ or retired $(26.4 \%$, $n=304)$. A majority: had type 2 diabetes $(63.2 \%, n=729)$; reported having diabetes for 11 years or longer $(52.6 \%, n=$ $607)$; had no episodes of hypoglycaemia $(78.1 \%, n=901)$; and, in terms of treatment, were using insulin $(49.6 \%, n=$ $572)$ or oral glucose-lowering agents $(28.1 \%, n=324)$. Finally, $54.9 \% \quad(n=601)$ of participants reported no diabetes-related complications, $24.7 \%(n=270)$ reported having one complication, and $20.4 \%(n=223)$ reported having two or more complications.

Procedure To identify a short-form version of the PAID, two subsamples were randomly created $(n=589$ and $n=564)$. No statistically significant differences between the subsamples were noted on the variables of age, sex composition, type of diabetes, glycosylated haemoglobin level, work status, complication frequency status and average WHO-5 scores. Thus, the random division produced comparable groups of participants.

\section{Results}

Five-item short form For subgroup 1, we conducted a principal-components analysis, which is an item-reduction technique, and found a one-component solution. Ten items loaded above 0.50 , all of which were from the Diabetesrelated Negative Emotions subscale (items 3, 6, 7, 8, 9, 10, $12,16,19,20)$. We removed three items rated on average by respondents as less than a 'minor problem' (items 8,10 , 20) and the remaining seven items were subjected to a reliability analysis. The two items which correlated most poorly with the other items were removed, resulting in a five-item short-form version (items 3, 6, 12, 16, 19-see Electronic Supplementary Material [ESM]). Cronbach's alpha, a measure of the reliability or correlation between items in a test, was good (see Table 1). Total scores on the PAID-5 can range from 0 to 20, with higher scores suggesting greater diabetes-related emotional distress. For subsample 1 , the average score was $6.07(\mathrm{SD}=5.09$; range 0-20).

The PAID-5 total score correlated significantly with the PAID-20 total score, $r=0.92, p<0.001$. The validity of the PAID-5 was also demonstrated by a statistically significant correlation with a measure of well-being (the WHO-5), $r=-0.47, p<0.001$. The direction of this correlation suggests that as diabetes-related distress increases, wellbeing decreases. Correlations between the PAID-5 and the other subscales of the PAID (treatment-related problems; food-related problems; lack of social support) were also investigated. Consistent with research suggesting that different aspects of diabetes-related distress are interrelated, 
Table 1 Scale score reliability for all measures

${ }^{a}$ Two-item measure; thus, a correlation coefficient was computed $(p<0.001)$

\begin{tabular}{lll}
\hline Measure & \multicolumn{2}{l}{ Cronbach's $\alpha(95 \% \mathrm{CI})$} \\
\cline { 2 - 3 } & Subsample 1 $(n=589)$ & Subsample 2 $(n=564)$ \\
\hline PAID-5 & $0.86(0.84-0.88)$ & $0.83(0.80-0.85)$ \\
PAID-Treatment problems & $0.67(0.63-0.72)$ & $0.66(0.60-0.70)$ \\
PAID-Food problems & $0.77(0.73-0.80)$ & $0.78(0.75-0.81)$ \\
PAID-Lack of social support & $r=0.54^{\mathrm{a}}$ & $r=0.66^{\mathrm{a}}$ \\
WHO-5 & $0.84(0.82-0.86)$ & $0.85(0.83-0.87)$ \\
\hline
\end{tabular}

yet distinct, the PAID-5 correlated positively with scores on the other problem areas: PAID-5/treatment, $r=0.64$; PAID-5/food, $r=0.61$; PAID-5/social support, $r=0.58$ (all $p$ values $<0.001)$. Finally, in accordance with previous research on sex differences in diabetes-related distress [7], we found that women had a significantly higher mean score than men (women's mean score $=6.81, \mathrm{SD}=5.27$; men's mean score $=5.23, \mathrm{SD}=4.74)$ on the PAID-5, $(t[587]=3.79$, $p<0.001$, Cohen's $d=0.32$ ).

The five items retained from the analyses conducted with subsample 1 were subjected to an exploratory factor analysis (EFA) using subsample 2. In this context, the EFA was used to identify the number of possible factors reflected in the correlations among the five items. It was anticipated that, as all items focused on diabetes-related distress, a single factor would emerge. The data were not normally distributed (i.e. scale items were positively skewed). Thus, principal-axis factoring, a specific type of EFA, was employed. A one-factor solution was found (eigenvalue $=2.97$ ), accounting for approximately $59.3 \%$ of the variance. Cronbach's $\alpha$ was good (see Table 1), and the mean score was $6.22(\mathrm{SD}=4.62$, range $=0-20)$.

As was observed with subsample 1, scores on the PAID-5 correlated negatively with scores on the WHO-5, $r=-0.40$, $p<0.001$. Scores on the PAID-5 and problems related to treatment, food, and social support also were found to correlate significantly: PAID-5/treatment, $r=0.60$; PAID-5/ food, $r=0.65$; and PAID-5/social support, $r=0.62$ (all $p$ values $<0.001)$. Again, female participants obtained higher scores on the PAID-5 than male participants (women: mean score $=7.14, \mathrm{SD}=4.93$; men: mean score $=5.27, S D=$ 4.06, $t[546.10]=4.94, p<0.001$, Cohen's $d=0.41$ ).

Diagnostic accuracy of the PAID-5 was established using receiver operating characteristic analysis with both subsamples. To evaluate the ability of the PAID-5 to 'diagnose' high distress, a cut-off score of $\geq 33$ on the 20 item PAID was used [5]. Using the optimal cut-off score of $\geq 8$ on the PAID-5, the AUC value was 0.97 (95\% CI $0.96-$ 0.98 ) in subsample 1 and 0.98 (95\% CI 0.97-0.99) in subsample 2 , while sensitivity was $95 \%$ and specificity was $89 \%$ for each of the subsamples.
Single-item screening measure Based on its psychometric properties, Item 12 ('Worrying about the future and the possibility of serious complications'), hereafter called PAID-1, appeared most suitable as a rapid screen for diabetes distress. For subsamples 1 and 2, scores on this item correlated significantly with the WHO-5, the 20 item PAID and with scores on the treatment, food, and social support subscales of the PAID. The same sex difference noted for the PAID-5 emerged, with women having significantly higher scores on this item in each subsample. When using a cut-off score of $\geq 33$ on the 20 item PAID (5), the optimal cut-off score of $\geq 3$ on the PAID-1 yielded AUC values of 0.87 (95\% CI $0.84-0.90)$ and $0.86(95 \%$ CI 0.82-0.89) for subsamples 1 and 2. Sensitivity and specificity values were (in order): $75 \%$ and $86 \%$ (subsample 1) and $74 \%$ and $86 \%$ (subsample 2 ).

\section{Discussion}

Our findings suggest that a five-item version of the PAID possesses good reliability and validity. The diagnostic accuracy of the scale was acceptable, achieving a sensitivity rate of $95 \%$ and a specificity rate of $89 \%$. The PAID-1 appears suitable for screening purposes in clinical settings, with adequate validity and a diagnostic accuracy of around $80 \%$. However, a high score on this item should prompt the clinician to administer the longer (and more reliable) PAID-5 (see ESM).

A major strength of the PAID-5 is that it takes less than 1 min to complete, yet it has comparable 'diagnostic' performance to the four-item Diabetes Distress Scale [9]. Both contain items assessing fear, depressed mood and the demands of living with diabetes; the PAID-1 focuses uniquely on concerns for the future, and this item is also included within the PAID-5.

Previous research has demonstrated that brief, verbally administered questions are effective at identifying depression in primary care [10]. Future research might seek to compare the effectiveness of verbal vs written administra- 
tion of the PAID-1, as well as its sensitivity to change following an intervention.

Additional psychometric testing of the PAID-5 and PAID-1 is required. However, the current study suggests that clinicians may now choose from more than one shortform measure of diabetes-related emotional distress, with their choice of scale likely to depend on local practice and previous experience.

Acknowledgements We are grateful to N. Zandbelt and W. Cleijne for their assistance in data management and to Novo Nordisk for an unrestricted research grant to conduct the DAWN MIND study.

Duality of interest The DAWN MIND study is supported by an unrestricted research grant from Novo Nordisk. D. Mathews has undertaken speaking engagements and research advisory boards with Novo Nordisk and the Oxford Centre for Diabetes, Endocrinology and Metabolism has a funding partnership with Novo Nordisk.

\section{References}

1. Centers for Disease Control and Prevention (2004) Serious psychological distress among persons with diabetes-New York City, 2003. Morb Mort Wkly Rep 53:1089-1092
2. International Diabetes Federation Clinical Guidelines Task Force (2005) Global guidelines for type 2 diabetes. International Diabetes Federation, Brussels

3. American Diabetes Association (2009) Standards of medical care in diabetes-2009. Diabetes Care 32(Suppl. 1):s13-s61

4. Polonsky WH, Anderson BJ, Lohrer PA et al (1995) Assessment of diabetes-related distress. Diabetes Care 18:754-760

5. Hermanns N, Kulzer B, Krichbaun M, Kubiak T, Haak T (2006) How to screen for depression and emotional problems in patients with diabetes: comparison of screening characteristics of depression questionnaires, measurement of diabetes-specific emotional problems and standard clinical assessment. Diabetologia 49:469-477

6. El Achhab Y, Nejjari C, Chikri M, Lyoussi B (2008) Diseasespecific health-related quality of life instruments among adult diabetics: a systematic review. Diabetes Res Clin Pract 80:171-184

7. Snoek FJ, Pouwer F, Welch GW, Polonsky WH (2006) Diabetesrelated emotional distress in Dutch and U.S. diabetic patients. Cross-cultural validity of the Problem Areas in Diabetes Scale (PAID). Diabetes Care 23:1305-1309

8. Fisher L, Glasgow RE, Mullan JT, Skaff MM, Polonsky WH (2008) Development of a brief diabetes distress screening instrument. Annals Family Med 6:246-252

9. Zandbelt N, Eldrup E, Gagliardino JJ et al. (2008) Well-being and glycemic control in diabetes patients: preliminary data from the cross-national DAWN Monitoring of Individual Needs in Diabetes (MIND) study. Diabetes 57(Suppl 1): 1906P, A529 (abstract)

10. Arrol B, Khin N, Kerse N (2003) Screening for depression in primary care with two verbally asked questions: cross sectional study. BMJ 327:1144-1146 